Rakenteiden Mekaniikka (Journal of Structural Mechanics)

Vol. 50, No 3, 2017, pp. 131-136

https://rakenteidenmekaniikka.journal.fi/index

https:/doi.org/10.23998/rm.65042

(c)Author(s) 2017.

Open access under CC BY-SA 4.0 license.

\title{
X-ray micro-tomography based FEM modelling of hygroexpansion in PLA composites reinforced with birch pulp fibers
}

\author{
Stefania Fortino ${ }^{1}$, Merja Sippola, Tom Andersson, Kirsi Immonen, Arttu Miettinen and \\ Petr Hradil
}

\begin{abstract}
Summary. This article presents a microscale modelling approach coupled with X-ray computed micro-tomography for the evaluation of material properties of polylactic acid (PLA) reinforced by birch pulp fibers under the effect of moisture. The results in terms of elastic moduli and hygroexpansion deformation were found in good agreement with the measurements taken at different levels of water uptake.
\end{abstract}

Key words: PLA composites, wooden fibers, hygroexpansion, X-ray micro-tomography, imagebased modelling, FEM.

Received 21 June 2017. Accepted 10 July 2017. Published online 21 August 2017.

\section{Introduction}

Currently the composite manufacturing in the construction and automotive sectors rely on engineering plastics and/or glass fiber reinforced composite materials that are nonsustainable and difficult to recycle. The most promising alternatives are bio-based materials such as wood plastic composites (WPCs) and natural fiber composites (NFCs) [1]. Compared to other fibrous materials, plant fibers are more suitable to reinforce plastics due to their relatively high strength and stiffness, low density, low $\mathrm{CO}_{2}$ emission and biodegradability. Forecast for 2020 by Nova Institute $\mathrm{GmbH}$ for ecology and innovation predicts a continuous production growth of these biocomposites [2].

In contrast to more commonly used glass fibers, wood fibers are characterized by variable sizes and anisotropic properties. Short fiber length in the final composite (typically $0.2 \mathrm{~mm}-0.8 \mathrm{~mm}$ ) often limits the strength of the material. Both earlier and current experimental research activities on biocomposites include development of fiber

\footnotetext{
${ }^{1}$ Corresponding author stefania.fortino@vtt.fi
} 
treatments and improvement of the fiber properties such as aspect ratio and fiber length. A relevant issue is that WPCs and NFCs absorb water into the naturally hydrophilic wood fibers embedded in the matrix. Variations of moisture content cause hygroexpansion and can produce moisture-induced creep in the final products. Poor bond of the fibers with the hydrophobic matrix induces moisture influence in these biocomposites with consequent strength reduction. In this context, deep understanding of microscale phenomena in biocomposites is crucial for the assessment of material properties. The experimental and computational research on the microstructure of biobased composites started to gain special interest in the last few years along with the development of multiscale modelling of materials [3,4]. In particular, image-based modelling has been introduced as a powerful tool to couple advanced experimental techniques (SEM, X-ray micro-tomography, etc.) with numerical simulations. The basic idea of this approach is to use the actual microstructure of heterogeneous materials, obtained by utilizing a suitable 2D or 3D imaging modality, as the geometric input for the models to assess, e.g., mechanical or transport properties of the material [5].

The present paper proposes a microscale modelling approach coupled with X-ray computed micro-tomography suitable for the evaluation of the material properties of polylactic acid (PLA) reinforced by birch pulp fibers under the effect of moisture.

\section{Water absorption and tensile tests of PLA composites}

Dog-bone specimens (Figure 1a) were prepared by using polylactic acid Natureworks Ingeo 3001D polymer with MFI 22 and birch pulp fibers provided by Metsä Fibre. Matrix and fibers were compounded with a twin-screw compounder and injection molded to the dog bone shaped tensile test specimen containing $40 \%$ fibers by weight according to ISO 527. Macroscale water absorption tests were carried out to measure the different levels of moisture uptake reached in the samples after 1 day, 7 days and 28 days immersion. At this end, a large sample for water absorption $(2 \mathrm{~cm} \times 2 \mathrm{~cm})$ was cut from the head of dog bone specimen (flange) to minimize the measurement error and the amount of cut edges. To consider only the water diffusion along z-direction, the lateral sides of the large sample, including cut surfaces, were protected by a foil. Measurement of water absorption was done according to ISO 62 standard from soaked samples (water at $23{ }^{\circ} \mathrm{C}$ ) starting from oven dried samples $\left(50{ }^{\circ} \mathrm{C}\right.$, min $24 \mathrm{~h}$ ). The moisture content was evaluated through the measurements of the sample weight changes. The dimensional changes of composites at different moisture content uptakes were also measured. It has to be noticed that in the specimen bar (web) the fibers are mainly oriented according to the resin flow while in the flange the fiber orientations are more random. Tensile tests were performed to evaluate the strength of the PLA composites at different levels of moisture contents. The used method was ISO 527 (5 $\mathrm{mm} / \mathrm{min}$ ). The tests measured the stress in the middle of the web. In order to get direct correlation between test samples and water absorption, the specimens were oven dried $\left(50{ }^{\circ} \mathrm{C}\right.$, min $24 \mathrm{~h}$ ), which was a deviation from the normal procedure where samples are conditioned at least 5 days in $23^{\circ} \mathrm{C}$ and $50 \%$ relative humidity before measurement. The immersed samples were measured immediately after immersion at 1, 7 and 28 days. 


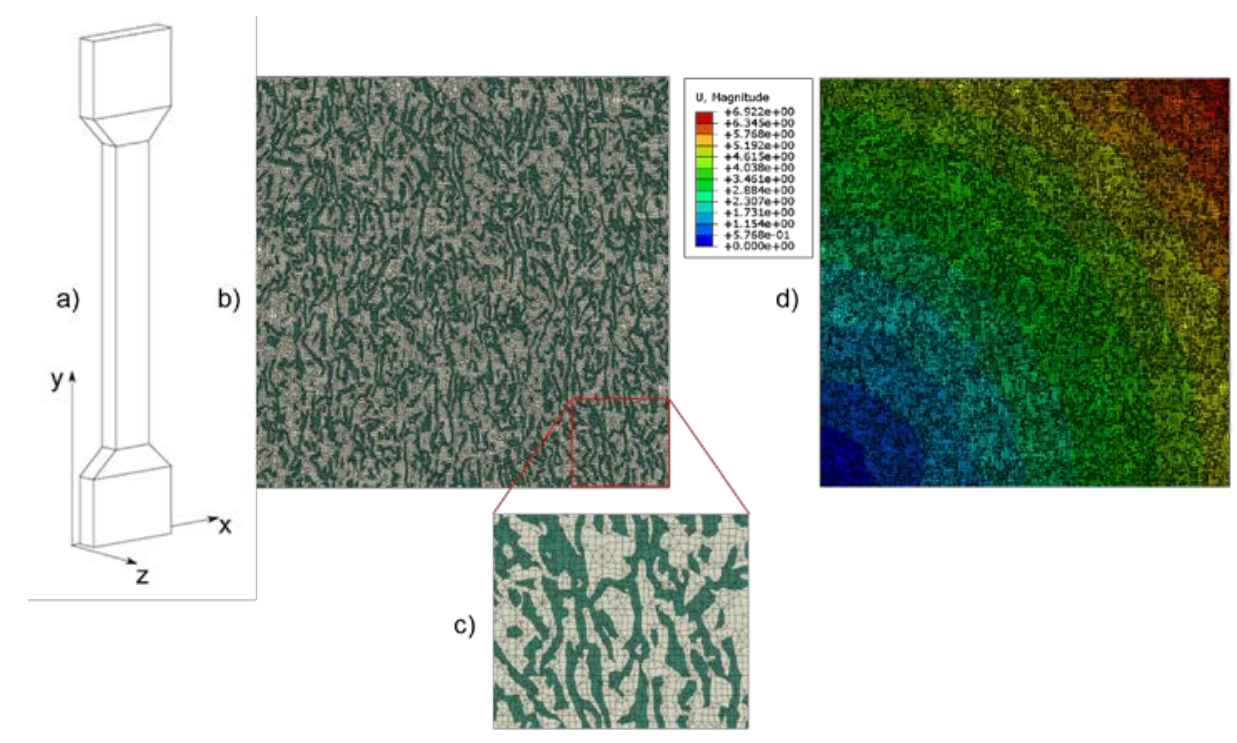

Figure 1. a) Scheme of the dog bone sample; b) FEM mesh (matrix in grey and fibers in green) and representative volume element (RVE) of the segmented tomography image in the xy plane (dry specimen), dimensions $1018 \mu \mathrm{m} \times 1401 \mu \mathrm{m}$; c) zoom of FEM mesh; d) displacement magnitude after 28 days of water immersion $(\mu \mathrm{m})$.

\section{Image-based modelling}

\section{$X$-ray tomography}

The multiscale tomography system available at University of Jyväskylä was used to capture tomographic images of the specimen. A cylindrical sample of approximately 2 $\mathrm{mm}$ diameter was cut from the middle web of the dry composite dog bone specimen by using a rotary tool. The sample was glued at the top of a sample holder rod and a microtomographic image was acquired using XRadia MicroXCT-400 device with $1.2 \mu \mathrm{m}$ resolution, $30 \mathrm{kV}$ acceleration voltage and $3 \mathrm{~W}$ X-ray power (see also [6]).

Two-dimensional FEM meshes were created from the micro-tomographic images by using the oof2 open source software after segmentation to a binary image with the open source code ImageJ. After the initial discretization in oof2, the FEM meshes were further processed and smoothened to improve mesh quality. The FEM mesh of segmented tomography image in the xy-plane is shown in Figure 1b. The microstructure model contains two material phases, the matrix and the fibers. In [7] the two-phase approach was found suitable enough for calculation of elastic moduli of PLA composites reinforced by birch pulp fibers compared to a more accurate three-phase approach including also the material at the interface between matrix and fibers.

\section{Microstructural FEM analyses}

Building up of FEM models, mesh modification steps and post-processing of results were carried out following the methods available within VTT ProperTune ${ }^{\mathrm{TM}}$ multiscale modelling toolset (see references in [7]). The two-dimensional microstructural FEM 
analysis of representative volume element (RVE) in xy-plane (Figure 1b) was carried out by using Abaqus code for different moisture contents. For details on the FEM modelling of bio-based composite microstructures the reader is referred to [7] where a PLA composite reinforced by birch pulp in dry conditions was studied. The assumption of plane stress state was found appropriate for the biocomposites with short fibers as discussed in [7]. In the same paper, the theoretical details related to the use of 2D RVEs for calculation of elastic modulus and Poisson ratio are discussed.

In the present paper the plane stress elements available in Abaqus were adopted (quadrilateral CPS4 and triangular CPS3). For the elasticity calculations, both matrix and fibers were assumed to be isotropic materials. The elastic moduli of fibers was assumed to be a parabolic function of moisture content starting from the reference value of $24 \mathrm{GPa}$ in dry state. Linear functions used in the previous literature for softwood [8] are not found suitable because of the hemicellulose softening in birch pulp fibers that provides a significant reduction of elastic moduli of PLA reinforced biocomposites during absorption tests. In Table 1 are reported the results obtained by the microstructural analysis of the RVE in the xy-plane.

Table 1. Values of moisture content changes $(\triangle M C)$ during the absorption test, elastic moduli of matrix $\left(E_{m}\right)$ and fiber $\left(E_{f}\right)$, Poisson ratios of matrix $\left(v_{m}\right)$ and fiber $\left(v_{f}\right)$, numerical Poisson ratios of composite $\left(v_{c, \text { num }}\right)$, numerical $\left(E_{c, \text { num }}\right)$ and measured $\left(E_{c, \text { meas }}\right)$ elastic moduli of composite.

\begin{tabular}{llllrllll}
\hline $\begin{array}{l}\text { water immersion } \\
\text { time }\end{array}$ & $\begin{array}{l}\Delta M C \\
(\%)\end{array}$ & $\begin{array}{l}E_{m} \\
(\mathrm{MPa})\end{array}$ & $v_{m}$ & $\begin{array}{l}E_{f} \\
(\mathrm{MPa})\end{array}$ & $v_{f}$ & $v_{c, \text { num }}$ & $\begin{array}{l}E_{c, \text { num }} \\
(\mathrm{MPa})\end{array}$ & $\begin{array}{l}E_{c, \text { meas }} \\
(\mathrm{MPa})\end{array}$ \\
\hline 1 day & 1.81 & 3327 & 0.42 & 22808 & 0.2 & 0.3817 & 6687 & 6812 \\
7 days & 4.59 & 3327 & 0.42 & 18790 & 0.2 & 0.3803 & 6216 & 6560 \\
28 days & 9.11 & 3327 & 0.42 & 6604 & 0.2 & 0.3737 & 4240 & 4368 \\
\hline
\end{tabular}

A simplified 2D FEM hygroexpansion analysis of the RVE in xy-plane was carried out to identify the values of the hygroexpansion coefficients suitable for the studied biocomposites. In Abaqus the hygroexpansion was simulated by assigning the moisture change to the whole body and hygroexpansion coefficients to the matrix as well as in the various directions of fibers. A Python script was built to determine the principal direction of fibers as the average of normal vectors of element faces over all elements in the fiber phase. As a simplified approach, this principal direction was assigned to the whole fiber phase as local material orientation in Abaqus analysis. The hygroexpansion coefficient of the biocomposite in direction $i$ is defined as $\alpha_{i}=\varepsilon_{\mathrm{h}, \mathrm{i}} / \Delta M C$ where $\Delta M C$ is the change in the moisture content and $\varepsilon_{\mathrm{h}, \mathrm{i}}=\left(L_{1, \mathrm{i}}-L_{0, \mathrm{i}}\right) / L_{0, \mathrm{i}}$ where $L_{1, \mathrm{i}}$ and $L_{0, \mathrm{i}}$ are the final and initial dimensions in that direction, respectively. The literature [9] shows a large range for the values of transversal hygroexpansion coefficients of fibers $\left(0.07 \leq \alpha_{\mathrm{T}}\right.$, fiber $\leq 0.30$ ). The values of longitudinal coefficients are around $\alpha_{\mathrm{L} \text {,fiber }}=\alpha_{\mathrm{T} \text {,fiber }} / 20$. In the present work $\alpha_{\mathrm{T} \text {,fiber }}=0.29, \alpha_{\mathrm{L} \text {,fiber }}=0.0145$ and $\alpha_{\mathrm{PLA}}=1 \mathrm{E}-4$ (see [4]) were found suitable to simulate the hygroexpansion of the studied biocomposites. The microstructural analysis provided a hygroexpansion deformation in the x-direction $\varepsilon_{\mathrm{h}, \mathrm{x}, \mathrm{num}}=0.008144$ that is practically equal to the measured $\varepsilon_{\mathrm{h}, \mathrm{x}, \text { meas }}=0.008142$ after 28 days of water absorption. Figure 1d shows the reached displacement magnitude. 


\section{Conclusions and future work}

A microscale modelling approach based on X-ray tomography was proposed for the evaluation of material properties of PLA composites reinforced by birch pulp fibers, under the effect of water content. Although only simplified two-dimensional FEM analyses were carried out, the results in terms of elastic moduli and hygroexpansion deformation were found in good agreement with the measurements taken at different levels of water uptake. Future basic research work is needed to develop accurate threedimensional models that will be more suitable for various types of fiber shapes and materials as well as for the fiber-matrix interactions under hygro-thermal effects.

\section{Acknowledgement}

The VTT projects UpTune and IndBio for developments of computational tools for biomaterials within VTT ProperTune ${ }^{\mathrm{TM}}$ toolset are gratefully acknowledged.

\section{References}

[1] A. Ashori. Wood-plastic composites as promising green-composites for automotive industries! Bioresource Technology 99(11), 4661-4667,2008. https://doi.org/10.1016/j.biortech.2007.09.043

[2] M. Carus, A. Eder, L. Dammer, H. Korte, L. Scholz, R. Essel, E. Breitmeyer. WPC/NFC Market study. Nova Institut-GmbH, Version 2014.10, 2014.

[3] A. Torgnysdotter, A. Kulachenko, P. Gradin, L. Wågberg. Fiber/fiber crosses: finite element modeling and comparison with experiment. Journal of Composite Materials 41(13), 1603-1618, 2007. https://doi.org/10.1177/0021998306069873

[4] T. Joffre. Structure and Mechanical Behaviour of Wood-Fiber composites. PhD Thesis, University of Uppsala, Faculty of Science and Engineering, 2014.

[5] A. Miettinen. Characterization of three-dimensional microstructure of composite materials by $X$-ray tomography, PhD thesis, Department of Physics, University of Jyväskylä, Finland, 2016.

[6] A. Miettinen, T. Harjupatana, M. Kataja, S. Fortino, K. Immonen. Time-resolved $\mathrm{X}$-ray microtomographic measurement of water transport in wood-fiber reinforced composite material. IOP Conference Series: Materials Science and Engineering, Volume 139, Number 1, 2016.

[7] M. Sippola, K. Immonen, A. Miettinen, A. Laukkanen, T. Andersson, H. Peltola, A. Harlin, K. Holmberg. Predicting stiffness and strength of birch pulp - polylactic acid composites. Journal of Composite Materials,50(18), 2549-2563 2015. https://doi.org/10.1177/0021998315608431

[8] M. Muzamal, E.K. Gamstedt, A. Rasmuson. Modeling wood fiber deformation caused by vapour expansion during steam explosion of wood. Wood Sci Technol 48, 353-372, 2014. https://doi.org/10.1007/s00226-013-0613-0 
[9] J. Strömbro, P. Gudmundson. An anisotropic fiber-network model for mechanosorptive creep in paper. International Journal of Solids and Structures 45, 57655787, 2008. https://doi.org/10.1016/j.ijsolstr.2008.06.010

Stefania Fortino, Merja Sippola, Tom Andersson, Kirsi Immonen, Petr Hradil VTT Technical Research Centre of Finland Ltd P.O. Box 1000, FI-02044 VTT, Finland stefania.fortino@vtt.fi

Arttu Miettinen

University of Jyväskylä, Department of Physics

P.O. Box 35, FI-40014 University of Jyväskylä, Finland

arttu.miettinen@phys.jyu.fi 